\title{
Controlling Ligand Surface Density on Streptavidin-Magnetic Particles by a Simple, Rapid, and Reliable Chemiluminescent Test
}

\author{
Diego Bouzas-Ramos, ${ }^{\dagger,+, \|}$ Laura Trapiella-Alfonso, ${ }^{\dagger, \S, \|}$ Kelly Pons, ${ }^{\dagger, \S}$ Jorge Ruiz Encinar, ${ }^{\ddagger \odot}$ \\ José M. Costa-Fernández, Vassilis Tsatsaris, ${ }^{\S}$ and Nathalie Gagey-Eilstein, ${ }^{*}, \pitchfork \odot$
}

${ }^{\dagger}$ UMR 8638 CNRS, Université Paris Descartes, Faculté des Sciences Pharmaceutiques et Biologiques, Sorbonne Paris Cité. 4 avenue de l'observatoire, 75006 Paris, France

${ }^{\ddagger}$ Department of Physical and Analytical Chemistry, Faculty of Chemistry, University of Oviedo, Avda. Julián Clavería, 8, 33006 Oviedo, Spain

${ }^{\S}$ Cochin Hospital, Assistance Publique-Hôpital de Paris, DHU Risques et Grossesse, Paris Descartes University, INSERM UMR 1139, PremUP Foundation, 53 Avenue de l'Observatoire, 75014 Paris, France

Supporting Information

ABSTRACT: The use of functionalized magnetic particles is increasing because they simplify the analytical process and yield promising results in a wide range of applications. Particularly, streptavidin-coated magnetic beads offer the possibility of rapid and very efficient grafting of biomolecules. Unfortunately, current methods to monitor and compute this grafting process are cumbersome and scarce. We describe herein a simple, rapid, and reliable chemiluminescent assay we have developed to check the grafting rate of functionalized magnetic beads. The power of the assay also relies on its ability to predict the amount of ligands required to obtain a precise grafting rate. In addition, results were correlated with a more general parameter in material functionalization characterization like surface ligand density. Finally, the assay was validated for a wide variety of biotinylated biomolecule sizes, ranging from small molecules (around $200 \mathrm{Da}$ ) to antibodies (around $150 \mathrm{kDa}$ ). This approach will allow a precise quantification and prediction of the functionalization of magnetic particles that is of enormous importance for quality control in many applications.

\section{INTRODUCTION}

Monitoring of grafting rate and ligand surface density is key to further bioapplications of functionalized particles. The number of molecules per particle is a vital piece of information because with it users will be able to design and monitor quantitative in vitro and in vivo experiments. ${ }^{1}$ This interest relies on the importance of these ligands in the final properties of the particles, such as stability, reactivity, and toxicity as well as in vivo (i.e., biodistribution, cellular uptake, clearance pathway, formation of the protein corona, etc.) or environmental behavior of the particles. $^{2-4}$ Ligand density and surface reactive functions are also used, among others, as quality control parameters in the development of nanotherapeutic products. ${ }^{5,6}$

Despite the relevance of this kind of study, little work has been devoted to this, and sometimes this information is missed or undervalued. Inductively coupled plasma mass spectrometry $(\mathrm{ICP}-\mathrm{MS})^{7-10}$ and optical emission spectrometry (ICPOES), ${ }^{11}$ nuclear magnetic resonance spectroscopy (NMR), ${ }^{12}$ infrared spectroscopy (IR), ${ }^{6}$ microscale thermogravimetry analysis (TGA), ${ }^{13}$ X-ray photoelectron spectroscopy (XPS), ${ }^{14}$ and colorimetric or fluorimetric assays ${ }^{8,15}$ have been employed so far to characterize the particle surface coverage. In parallel, modeling approaches or theoretical calculations have been also developed either to confirm the experimental results or to predict the behavior and properties of the particle by modifying ligand density. ${ }^{16,17}$ Nevertheless, some of these strategies require sophisticated and expensive instrumentation, specialized users, and hard data treatment processes. Thus, simple, easy to use, and general approaches to access this important information are currently in high demand.

Regarding the specific biotin-streptavidin interaction, the strongest noncovalent interaction that exists, a few approaches have been developed to characterize the biotin-binding capacity of streptavidin-coated supports. ${ }^{18-20}$ All of these studies are focused on the total number of available binding sites on the support instead of on the grafting rate or the saturation rate by a given molecule. Finally, it should be noted that no general approach has been developed to evaluate and

Received: May 18, 2018

Revised: July 9, 2018

Published: July 10, 2018 


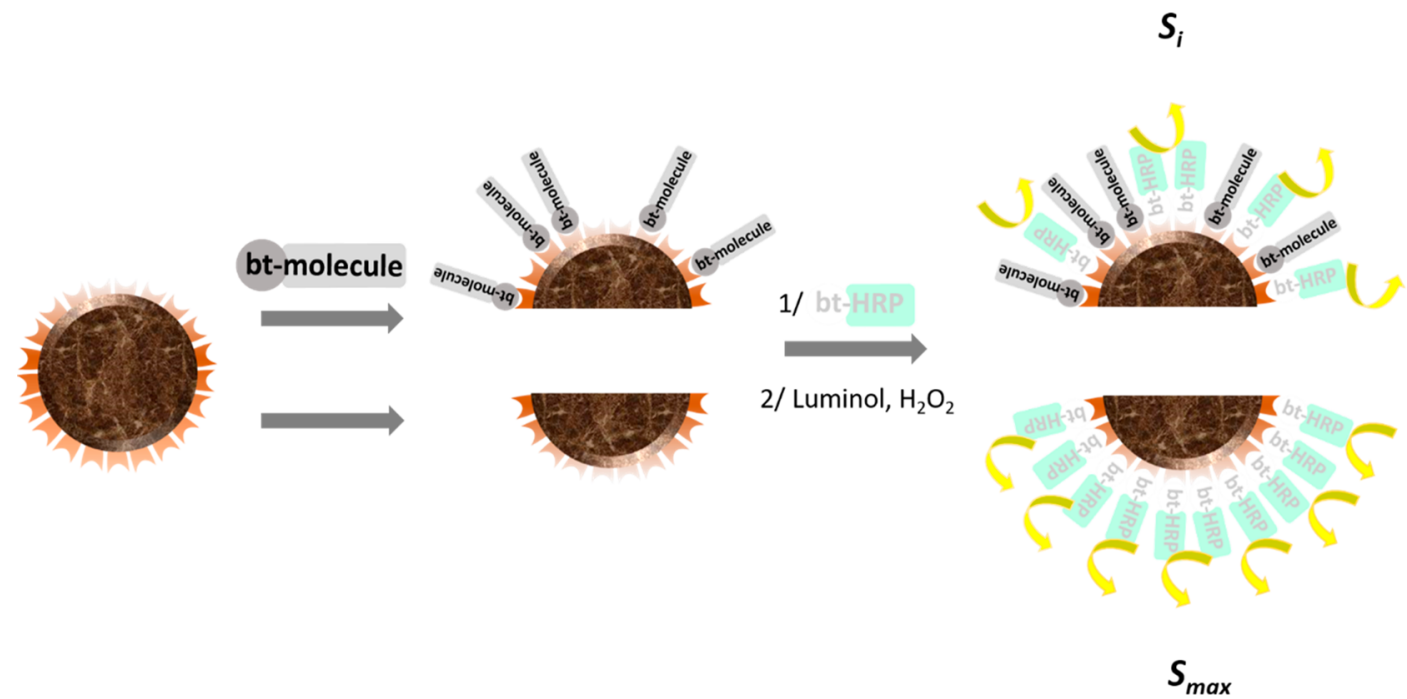

Figure 1. Schematic representation of the streptavidin MB grafting process and the bt-HRP chemiluminescent test.

monitor the grafting rate of a streptavidin-coated support applicable to a wide range of biotinylated ligand sizes.

Herein, we propose a rapid, simple, low-sample consumption and reliable chemiluminescent test to predict and monitor the grafting rates of streptavidin-magnetic beads $(\mathrm{MB})$. We have correlated our results with a more general characterization parameter such as the ligand surface density. MBs are selected as a model support since their use in bioapplications is nowadays increasing because they simplify the analytical process (e.g., purification steps, time of analysis, etc.), and yield promising results in a wide range of applications. ${ }^{21,22}$

The robustness of the proposed methodology has been validated with a range of biotinylated biomolecules with different molecular weights and also tested for its predictive power by grafting with unknown biotinylated proteins.

\section{RESULTS AND DISCUSSION}

Development and Optimization of bt-HRP Assay. Saturation rate and the number of ligands per particle are critical parameters for the correct design of magnetic particlebased quantitative bioassays. Thus, taking advantage of the use of streptavidin functionalized $\mathrm{MB}$, we have developed an indirect bioassay based on the measurement of free streptavidin sites on the beads after conjugation of the target biotinylated molecules (Figure 1). To do so, biotinylated horseradish peroxidase (bt-HRP) was used, and the subsequent addition of its substrate (luminol $+\mathrm{H}_{2} \mathrm{O}_{2}$ ) allowed quantification of the free streptavidin sites by chemiluminescence. Nongrafted beads must be used as a reference of the total sites available on the beads.

First of all, some operational conditions should be optimized. The amount of beads needed to perform the assays and the concentration of bt-HRP are related and must be optimized to ensure the maximum signal of the assay while reducing consumption of reagents. In optimizing the amount of beads, it is also crucial to adapt the protocol for easy handling of the MB by the final users. $20 \mu \mathrm{L}$ of beads and 100 $\mu \mathrm{L}$ of bt-HRP were selected under working conditions. Several dilutions of the stock solution of $\mathrm{MB}(10 \mathrm{mg} / \mathrm{mL})$ were tested, ranging from $1: 10$ to $1: 100$. Finally, a $1: 40$ dilution was selected as the best compromise between easy handling and bead consumption. More dilute solutions are difficult to handle, inducing a loss of beads during the washing steps, while more concentrated solutions require a large amount of beads and may disrupt signal measurement. Finally, the selected 1:40 dilution resulted in a consumption of $5 \mu \mathrm{g}$ of beads per well.

Maximum signal was then optimized by adjusting the concentration of bt-HRP. For this purpose, we built a saturation curve of bt-HRP (0 to $365 \mathrm{pmol} \mathrm{bt-HRP/mg}$ beads; Figure 2). As observed, a minimum bt-HRP

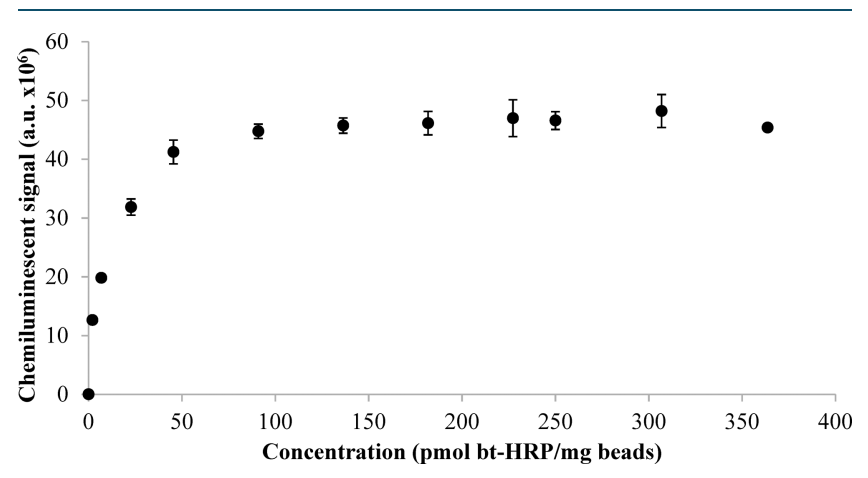

Figure 2. Saturation curve of streptavidin MB with bt-HRP.

concentration of $90 \mathrm{pmol} / \mathrm{mg}$ of beads ( $4.5 \mathrm{nM}$; inflection point of the curve) is required to saturate the beads. In this sense, to ensure the complete saturation of beads and a constant maximum signal (no depletion in bt-HRP in any conditions), we decided to work with a 3-times more concentrated solution of bt-HRP (300 pmol/mg, $15 \mathrm{nM})$.

Once these parameters were fixed, we evaluated the influence of incubation time. Thus, we saturated beads with bt-HRP at four different incubation times: 30, 60, 90, and 120 min. Although no significant differences in the chemiluminescent signal were observed, the shorter incubation times led to a less precise signal with a $\mathrm{CV}$ of $8 \%$, while for the rest of the assayed times the CV remained constant at $4 \%$. Therefore, $1 \mathrm{~h}$ was selected as the minimum incubation time required for a robust maximum signal (data not shown).

Grafting Yield and Stability. In order to achieve a precise amount of biomolecules grafted on the beads, the yield of grafting of $\mathrm{MB}$ with various molecules was evaluated. For this purpose, consecutive grafting procedures of $\mathrm{MB}$ with 
biotinylated molecules were carried out. After the first grafting, the supernatant was recovered and used again in a fresh $\mathrm{MB}$ suspension. This allows the molecules that have not been bound during the first grafting procedure to graft during the second grafting procedure. The saturation rate of $\mathrm{MB}$ of each step was estimated with the bt-HRP assay.

Figure 3 shows percentages of saturation after the two $\mathrm{MB}$ grafting procedures for three model molecules (biotin, bt-

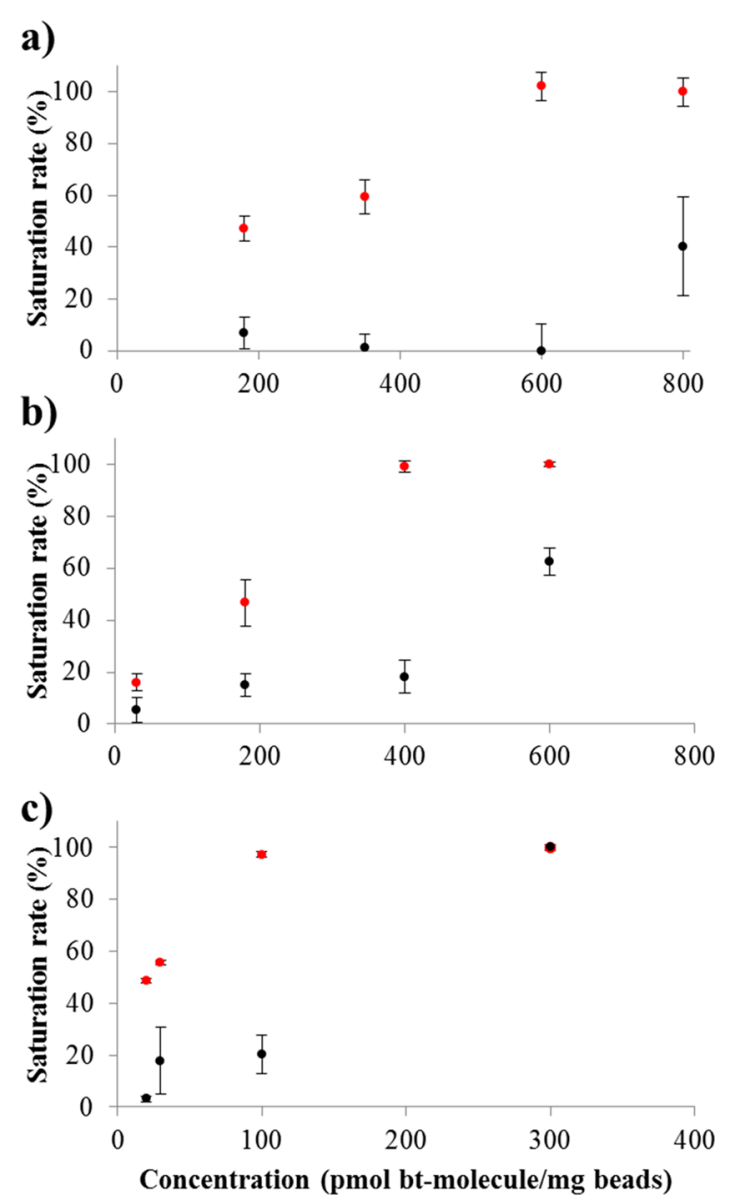

Figure 3. Saturation rate (\%) obtained after grafting (red points) and second grafting (black points) of $\mathrm{MB}$ with different concentrations of (a) biotin, (b) bt-VEGF 95 , and (c) bt-BSA. $n=4$, uncertainties correspond to $1 \mathrm{SD}$.

VEGF $_{95}$ and bt-BSA) at different concentrations. As observed in Figure 3a, before reaching the total saturation of beads (600 $\mathrm{pmol}$ biotin/mg of beads), biotin molecules were totally bound to the MB during the first grafting. Indeed, the saturation rate was negligible after the second grafting procedure. It is interesting to note that with $800 \mathrm{pmol}$ of biotin, corresponding to $200 \mathrm{pmol}$ above saturation, the resulting second saturation rate $(40 \%)$ was the same as the saturation rate obtained with 200 pmol of biotin in the first grafting, indicating a yield of grafting of $100 \%$.

In the case of bigger molecules, such as bt-VEGF ${ }_{95}(\mathrm{MW}=$ $28 \mathrm{kDa}$, Figure $3 \mathrm{~b}$ ) and bt-BSA ( $\mathrm{MW}=66 \mathrm{kDa}$, Figure $3 \mathrm{c}$ ), a small amount of ligand remained unbound after the first grafting as indicated by the small percentage of saturation observed after the second grafting at concentrations below total saturation. For example, at $200 \mathrm{pmol} / \mathrm{mg}$ of beads of btVEGF $_{95}$, a $15 \%$ saturation rate was obtained during the second grafting. The same behavior was observed for bt-BSA. Thus, for bt-VEGF ${ }_{95}$ and bt-BSA, the estimated yield of the grafting of MB was around $80 \%$. The observed difference in terms of yield of grafting between biotin and bigger biotinylated molecules may be due to biotin accessibility. We can assume that biotin covalently bound to a folded protein may be less available because of steric hindrance.

On the other hand, we evaluated the possibility of displacement of the biotin or another biotinylated molecule grafted onto MB by bt-HRP molecules during the incubation of grafted beads. For that purpose, a bt-HRP assay was carried out in both nongrafted $\mathrm{MB}$ and biotin-saturated MB (900 pmol biotin/mg beads) using a range of different concentrations of bt-HRP (from $0.05 \mathrm{nM}$ to $18 \mathrm{nM}$ ). As observed in Figure S-1 (see Supporting Information), biotin-saturated beads exhibited the same behavior at any bt-HRP concentration, with a mean saturated rate of $100 \%$, showing CV below $4 \%$. Additionally, the saturated rate of $\mathrm{MB}$ grafted with biotin at different concentrations (from 0 to $900 \mathrm{pmol}$ biotin/mg beads) was assessed at four different bt-HRP concentrations $(2.5,5,10$, and $15 \mathrm{nM}$, Figure S-2). As observed, there were no differences in the saturation obtained for each biotin-grafted $\mathrm{MB}$ with regard to bt-HRP concentrations used for incubation. Therefore, taking all of the above into account, we corroborated that no displacement of grafted molecules onto beads takes place during the 1-h incubation with bt-HRP, even at a high concentration $(15 \mathrm{nM})$. These results are consistent with the high-affinity constant of the streptavidin-biotin interaction. $^{23}$

Bead Saturation and Molecular Weights. Once yield of grafting was determined and assay robustness regarding displacement by bt-HRP was assessed, the grafting of $\mathrm{MB}$ with different biotinylated molecules at different molecular weights was evaluated. The saturated rate obtained by the assay was correlated with ligand surface density.

First, a set of different biotinylated molecules that covered a wide range of molecular weights was selected to assess the grafting of $\mathrm{MB}$. In addition, these molecules also present different chemical structures (i.e., small organic molecules, peptides, proteins, and antibodies). Biotin (244 Da) was selected as the smallest molecule since free biotin molecules should occupy all available streptavidin sites on the surface of the $\mathrm{MB}$ under saturating conditions, whereas a biotinylated antibody of $150 \mathrm{kDa}$ (immunoglobulin G, IgG) was selected as the biggest molecule to be evaluated. Different biotinylated molecules such as bt-peptide A (1951 Da), bt-VEGF 95 (28 000 $\mathrm{Da}), \mathrm{bt}^{-\mathrm{VEGF}_{165}}(40000 \mathrm{Da})$, and bt-BSA (66 $\left.000 \mathrm{Da}\right)$ were also used to explore the predictive power of the bt-HRP assay.

Figure S-3 displays the saturated rate of grafted MB for different biotinylated molecules over a wide range of concentrations ( 0 to $1000 \mathrm{pmol}$ molecule/mg beads). As can be observed, $100 \% \mathrm{MB}$ saturation was reached at different concentrations depending on the biotinylated molecule and, particularly, depending on its MW. Specifically, the saturated rate profile for all biotinylated molecules exhibits two welldifferentiated ranges: one range of linearity in which the saturation of $\mathrm{MB}$ increases as a function of the concentration of the molecule up to $100 \%$ saturation and another flat and constant range in which the concentration of molecules used for grafting leads to the total saturation of beads (Figures 4 and $\mathrm{S}-4)$. The concentration range assayed in the bt-HRP assay, the saturation concentration, as well as the linear range for each biotinylated molecule are all given in Table 1 . 

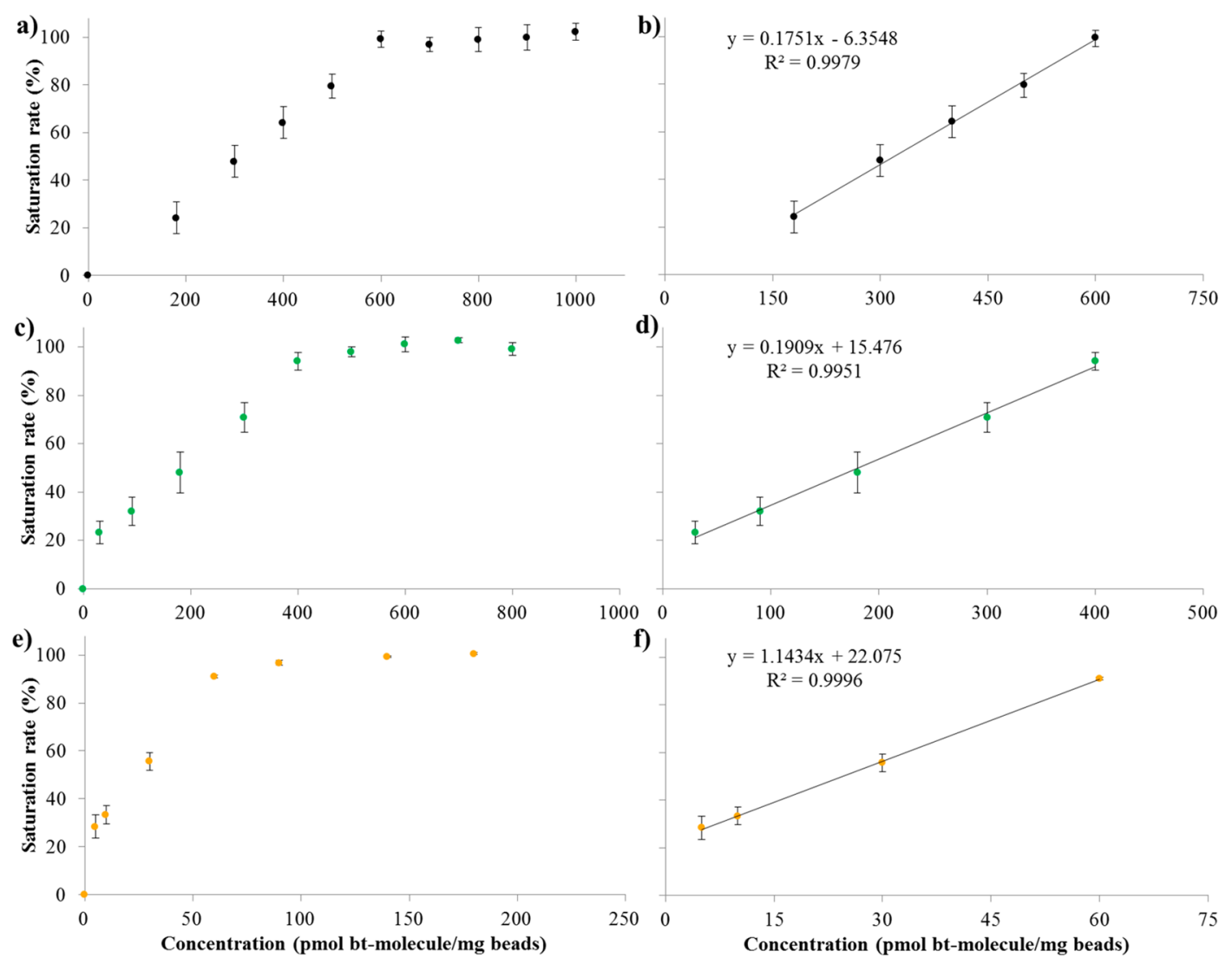

Figure 4. Saturation curves $(\mathrm{a}, \mathrm{c}, \mathrm{e})$ and linear relationships $(\mathrm{b}, \mathrm{d}, \mathrm{f})$ of the grafted MB at different concentrations of biotinylated molecules: (a,b) biotin (black); (c,d) bt-VEGF 95 (green); and (e,f) bt-goat IgG (orange). $n=3$, uncertainties correspond to 1 SD (continues in Figure S-4 in the Supporting Information).

Table 1. Concentration Range Assayed, Linear Range, and Saturation Concentration Obtained in the bt-HRP Assay for Various Biotinylated Molecules with Different Molecular Weights $(n=3$, Uncertainties Correspond to $1 \mathrm{SD})$

$\begin{array}{lcccc}\begin{array}{c}\text { biotinylated } \\ \text { molecule }\end{array} & \begin{array}{c}\mathrm{MW} \\ (\mathrm{kDa})\end{array} & \begin{array}{c}\text { concentration } \\ \text { range assayed } \\ \text { (pmol/mg beads) }\end{array} & \begin{array}{c}\text { linear range } \\ \text { (pmol/mg } \\ \text { beads) }\end{array} & \begin{array}{c}\text { saturation } \\ \text { concentration } \\ \text { (pmol/mg } \\ \text { beads) }\end{array} \\ \text { biotin } & 0.244 & 0-1000 & 180-600 & 605 \pm 10 \\ \text { bt-peptide A } & 1.951 & 0-900 & 90-600 & 625 \pm 15 \\ \text { bt-VEGF }_{95} & 28 & 0-800 & 30-400 & 440 \pm 15 \\ \text { bt-VEGF }_{165} & 40 & 0-500 & 10-180 & 190 \pm 5 \\ \text { bt-BSA } & 66 & 0-600 & 30-90 & 100 \pm 5 \\ \text { bt-goat IgG } & 150 & 0-180 & 5-60 & 70 \pm 2\end{array}$

As can be observed, the saturation of $\mathrm{MB}$ using the smallest molecule (biotin) was obtained using a concentration of $605 \pm$ $10 \mathrm{pmol}$ biotin/mg beads, while saturation with the biggest molecule (bt-goat $\operatorname{IgG}$ ) was obtained with a concentration of $70 \pm 2$ pmol bt-goat IgG/mg beads. Interestingly, these results are in perfect agreement with those reported for typical binding capacities of $1 \mathrm{mg}$ of commercial MB: ${ }^{24}$ between 650 and $900 \mathrm{pmol} / \mathrm{mg}$ for free biotin and $\sim 10 \mu \mathrm{g} / \mathrm{mg}(\sim 67 \mathrm{pmol} /$ $\mathrm{mg}$ ) for a biotinylated antibody.

Results obtained for all the biotinylated molecules assayed, whose MW range covers a wide interval from 0.2 to $150 \mathrm{kDa}$, seem to indicate that the saturation concentration is correlated with MW (Figures 4 and S-4, and Table 1). In other words, the amount of biotinylated molecule necessary to saturate $\mathrm{MB}$ decreases as the MW of the molecule increases, likely due to steric hindrance effects.

Determination of the Ligand Surface Density on Streptavidin-Magnetic Beads. The proposed bt-HRP assay evaluates behavior and saturated rate when grafting $\mathrm{MB}$. However, the percentage saturation of beads is a relative parameter. The ligand surface density $(\sigma)$ is a stronger factor as it is an absolute parameter. Such ligand surface density on the $\mathrm{MB}$ was determined using eq 2, which is based on the equation already reported by Elzey et al. ${ }^{11}$ and Ndolomingo et al., ${ }^{15}$ considering that yield of grafting is $100 \%$ :

$$
\sigma_{\text {calc }}=\left(\frac{m_{\text {molecule }}}{m_{\text {bead }}}\right)\left(\frac{N_{\mathrm{A}} \rho_{\text {bead }} d_{\text {bead }}}{6 \mathrm{MW}_{\text {molecule }}}\right)
$$

where $\sigma_{\text {calc }}$ is the calculated ligand surface density, $m_{\text {molecule }}$ and $m_{\text {bead }}$ are the biotinylated molecule mass and the magnetic bead mass, respectively, $N_{\mathrm{A}}$ is the Avogadro constant, $\rho_{\text {bead }}$ and $d_{\text {bead }}$ are the density and diameter of the spherical $M B$, respectively, and $\mathrm{MW}_{\text {molecule }}$ is the molecular weight of the biotinylated molecule used in the grafting.

Consequently, we were able to establish the correlation between the ligand surface density and the saturated rate of these grafted beads. In this context, Figure S-5 shows linear relationships that relate the saturated rate of beads (up to $100 \%$ bead saturation) with the ligand surface density for different concentrations of each biotinylated molecule. Furthermore, the ligand surface density at $100 \%$ saturation of beads was calculated for free biotin and the five biotinylated molecules. Ligand surface densities obtained in terms of 
number of molecules $/ \mu \mathrm{m}^{2}$ were the following: $266 \times 10^{3} \pm 4$ $\times 10^{3}$ for biotin $(244 \mathrm{Da}), 234 \times 10^{3} \pm 6 \times 10^{3}$ for bt-peptide A $(1951 \mathrm{Da}), 169 \times 10^{3} \pm 6 \times 10^{3}$ for bt-VEGF $95(28 \mathrm{kDa})$, $76 \times 10^{3} \pm 2 \times 10^{3}$ for bt-VEGF $165(40 \mathrm{kDa}), 38 \times 10^{3} \pm 2 \times$ $10^{3}$ for bt-BSA $(66 \mathrm{kDa})$, and $27 \times 10^{3} \pm 4 \times 10^{3}$ for bt-goat $\operatorname{IgG}(150 \mathrm{kDa})$. These results not only evidence the different surface packing of molecules but also confirm the fact that the ligand surface density on $\mathrm{MB}$ decreases as the MW of biotinylated molecules increases, leading to steric saturation of beads at greater molecular size. It is also interesting to note that the relation between ligand surface density and MW is not linear (see Figure S-6 in the Supporting Information), which confirms the importance of the volume occupied by the molecule and consequently steric hindrance.

Control and Validation of the Developed Methodology. In previous sections, we demonstrated that our bt-HRP assay establishes linear relationships between the saturated rate of $\mathrm{MB}$ or ligand surface density and the concentrations of biotinylated molecules used for the grafting of beads. In this context, it would be interesting to verify if those linear relationships can be used to predict the saturated rate of grafted beads with any other biotinylated molecules within the molecular range assayed.

In a first step, two other biotinylated molecules, a bt-peptide (bt-peptide B) of $1895 \mathrm{Da}$ and a bt-IgG (bt-antihuman endoglin IgG) of $150 \mathrm{kDa}$, were selected. The idea was to prove that we are able to estimate the concentration needed to obtain a desired saturation of beads based on the previous experimental results obtained for similar molecules (respectively, bt-peptide A and bt-goat IgG). Figures $4 \mathrm{f}$ and S-4b were respectively used to determine the required concentrations of each molecule to obtain $30 \%, 50 \%$, and $100 \%$ saturation of MB.

Table 2 gives the interpolated and experimental saturation results at different concentrations. As can be seen, the

Table 2. Saturation Rate (\%) Intended and Experimentally Obtained for Two Biotinylated Molecules (bt-peptide B and bt-anti-human endoglin IgG) Using the bt-HRP Assay and Concentrations Interpolated by Means of Equations of Linear Relationships Obtained for bt-peptide A (1951 Da) and bt-goat IgG $(150 \mathrm{kDa})$, respectively $(n=3$, Uncertainties Correspond to $1 \mathrm{SD}$ )

$\begin{array}{ccccc}\begin{array}{c}\text { Biotinylated } \\ \text { molecule }\end{array} & \begin{array}{c}\mathrm{MW} \\ (\mathrm{kDa})\end{array} & \begin{array}{c}\text { concentration } \\ \text { interpolated }^{a} \\ \text { (pmol/mg beads) }\end{array} & \begin{array}{c}\text { saturated } \\ \text { rate } \\ \text { intended } \\ (\%)\end{array} & \begin{array}{c}\text { saturated } \\ \text { rate } \\ \text { obtained } \\ (\%)\end{array} \\ \text { bt-peptide B } & 1.895 & 160 & 30 & 34 \pm 7 \\ & & 295 & 50 & 58 \pm 4 \\ \text { bt-IgG (bt- } & 150 & 625 & 100 & 98 \pm 1 \\ \text { antihuman } & & 5 & 30 & 26 \pm 4 \\ \text { endoglin IgG) } & & 25 & 50 & 52 \pm 4 \\ & & 70 & 100 & 99 \pm 1\end{array}$

${ }^{a}$ Equation used for bt-peptide B: saturated rate $=0.1496 \times[\mathrm{bt}-$ peptide $]+6.135$. Equation used for bt-antihuman endoglin IgG: saturated rate $=1.1434 \times[\mathrm{bt}-\mathrm{IgG}]+22.075$.

saturated rate given experimentally by the bt-HRP assay for both biotinylated molecules fits very well with the saturation percentages interpolated from the linear equations for each MW.

In a second step, we estimated the robustness of our predictive assay with a biotinylated molecule with any MW between 0.2 and $150 \mathrm{kDa}$. Using results from Figures 4 and S4, a correlation between concentration of biotinylated molecules and MW can be established. Specifically, two linear relationships $\left(C_{\text {molecule }}\right.$ function of $\mathrm{MW}_{\text {molecule }}$ ) for achieving half-saturation $(50 \%)$ and saturation $(100 \%)$ of the MB were obtained (Figure 5). An adequate correlation coefficient is obtained in the range between biotin (244 Da) and bt-BSA (66 $\mathrm{kDa})$. The bt-goat IgG $(150 \mathrm{kDa})$ was excluded due to its huge difference in MW in comparison with the other biotinylated molecules and bt-HRP. However, the linear relationship of the bt-goat IgG (Figure 4f) could be used to determine the necessary concentration of any bt-antibody to obtain the desired saturation of beads with this type of molecule.

In order to validate the potential of these linear correlations, two biotinylated molecules were assessed: a small bt-polymer $(844 \mathrm{Da})$ and the bt-TNF $\alpha$ protein $(51 \mathrm{kDa})$. In this way, the concentrations required to achieve $50 \%$ and $100 \%$ saturation with these two molecules could be easily predicted by inserting their corresponding molecular weights in the previously obtained equations (Figure 5). As shown in Table 3, the feasibility of this methodology was demonstrated, as the saturated rates obtained experimentally using the bt-HRP assay with the calculated concentrations were statistically indistinguishable from the saturation percentages intended, that is, $50 \%$ and $100 \%$.

Finally, a biotinylated single-stranded oligonucleotide of 80 nucleotides $(\sim 25 \mathrm{kDa})$ was also evaluated. Again, btoligonucleotide concentrations required to obtain $50 \%$ and $100 \%$ saturation were determined (185 and $410 \mathrm{pmol} / \mathrm{mg}$ beads, respectively). However, the saturation percentages obtained experimentally (Table 3 ) were both around $100 \%$. In this case, experimental and intended values were not in agreement with the predictive values of the assay. Nevertheless, these results are corroborated by the binding capacity announced in the commercial datasheet $(200 \mathrm{pmol} / \mathrm{mg}$ beads) ${ }^{24}$ showing the performance of the test in characterizing the grafting process. This particular behavior may be explained by the fact that DNA is a highly negatively charged molecule which can induce additional repulsion on the surface of the MB. It has also been reported that the binding capacity for oligonucleotides is inversely related to their molecular size (number of bases) and is reduced for large DNA fragments. ${ }^{25}$

\section{CONCLUSIONS}

In response to the need to monitor particle functionalization in terms of stoichiometry, saturation, and ligand surface density, we present a new rapid, easy, and cost-effective assay for the determination of $\mathrm{MB}$ saturation rate, which can be easily correlated with a more general characterization parameter such as surface ligand density. In order to fully characterize the functionalized particle, it is also recommended to evaluate ligand functionality after grafting by a complementary test (e.g., immunoassay). Note that both parameters (ligand density and ligand function) are related, since saturation rate may influence ligand functionality (i.e., crowded ligand at the surface may have a negative impact on its functionality). Of course, such a relationship should be studied for each specific case as it is species-specific. However, it should be mentioned here that there are many examples in the literature where such functionalized particles are grafted with different molecules while keeping their activity. ${ }^{26}$

In addition to this control issue, we should emphasize the remarkable predictive power of our assay that allows easily to 


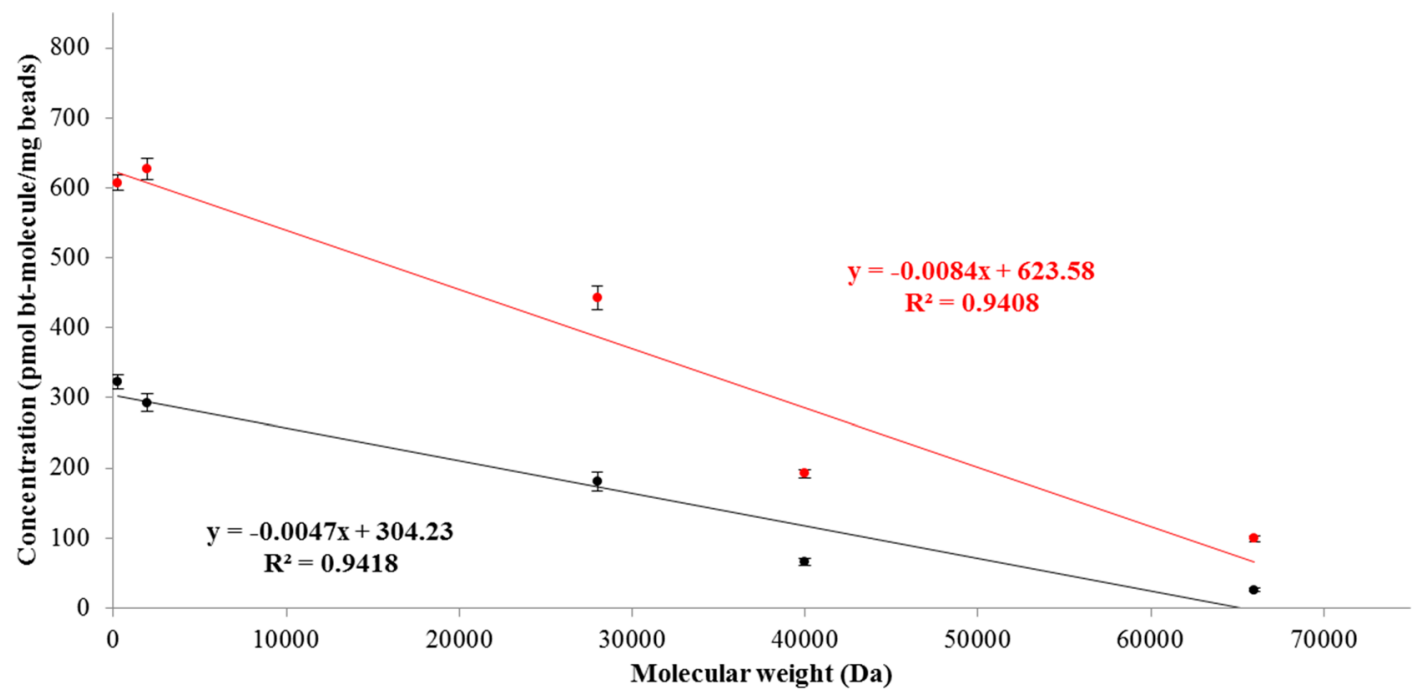

Figure 5. Linear relationship obtained when the influence of the molecular weight of the molecule used for the bead grafting on the concentration of the biotinylated molecule was studied to achieve $50 \%$ (black) and $100 \%$ (red) bead saturation. $n=3$, uncertainty corresponds to 1 SD.

Table 3. Saturation Rate (\%) Intended and Experimentally Obtained for Three Biotinylated Molecules (bt-polymer, bt-TNF $\alpha$ Protein, and bt-oligonucleotide) Using the bt-HRP Assay and Concentrations Interpolated by Means of Equations of Linear Relationships Relating the Molecular Weight (MW) of Biotinylated Molecules with Concentrations of These Molecules in Achieving 50\% and $100 \%$ Bead Saturation ( $n=3$, Uncertainties Correspond to 1 SD)

\begin{tabular}{|c|c|c|c|c|}
\hline biotinylated molecule & $\mathrm{MW}(\mathrm{kDa})$ & concentration interpolated $^{a}(\mathrm{pmol} / \mathrm{mg}$ beads $)$ & saturated rate intended (\%) & saturated rate obtained (\%) \\
\hline \multirow[t]{2}{*}{ bt-polymer } & 0.844 & 300 & 50 & $51 \pm 6$ \\
\hline & & 615 & 100 & $96 \pm 7$ \\
\hline \multirow[t]{2}{*}{ bt-TNF $\alpha$ protein } & 51 & 65 & 50 & $48 \pm 14$ \\
\hline & & 195 & 100 & $102 \pm 4$ \\
\hline \multirow[t]{2}{*}{ bt-oligonucleotide } & $\sim 25$ (80 nucleotides) & 185 & 50 & $100 \pm 1$ \\
\hline & & 410 & 100 & $100 \pm 2$ \\
\hline
\end{tabular}

${ }^{a}$ Equation used in achieving 50\% bead saturation: [bt-molecule] $=-0.0047 \times \mathrm{MW}+304.23$. Equation used in achieving $100 \%$ bead saturation: $[$ bt-molecule $]=-0.0084 \times \mathrm{MW}+623.58$.

control the bead grafting with any biotinylated molecule in a molecular range from 1 to $150 \mathrm{kDa}$, except for the special case of DNA. These control and predictive features will help users to efficiently design functionalized beads for further applications. Nevertheless, the predictive model was built using small molecules, linear peptides, and polymers and globular proteins (antibodies, enzymes...). Complementary studies with different types of biotinylated molecules (e.g., fibrillar proteins) could be performed in order to further generalize the predictive value of the assay.

Finally, our assay protocol can be considered as a general approach for the saturation rate determination of any streptavidin functionalized particle or support by adapting the protocol. For example, centrifugation or ultrafiltration can be employed as purification steps when nonmagnetic particles are used. If nanoparticles show native optical properties, spectral interference (energy transfer, spectral overlapping, etc.) should be considered and the methodology accordingly modified (e.g., change of enzyme substrate).

\section{MATERIALS AND METHODS}

Chemicals and Reagents. Magnetic beads Dynabeads M280 Streptavidin and magnetic supports DynaMag-5 Magnet and DynaMag-96 Side Skirted Magnet were from Invitrogen by Thermo Fisher Scientific (Eugene, OR, USA). Corning Costar 96-well White assay plates (high binding and polystyrene flat bottom) were from Sigma-Aldrich (SaintQuentin Fallavier, France).

Bovine serum albumin fraction IV (BSA) and Dulbecco's phosphate-buffered saline (PBS) were from Eurobio (Les Ulis, France). Tween 20 and 4-nitrophenyl phosphate disodium salt hexahydrate (pNPP) were from Sigma-Aldrich. Biotinylated peroxidase (bt-HRP) and Super Signal West Pico Chemiluminescent Substrate (containing stable peroxide solution and Luminol Enhancer solution) were from Thermo Fisher Scientific.

Biotin and N-biotinyl-NH(PEG) $)_{11}-\mathrm{COOH}$ (bt-polymer) were from Sigma-Aldrich. Biotinylated TNF $\alpha$ (bt-TNF $\alpha$ ), biotinylated VEGF $_{165}($ bt-VEGF 165$)$, biotinylated goat IgG (btgoat IgG), and biotinylated antihuman endoglin/CD105 antibody (bt-antihuman endoglin IgG) were from R\&D Systems (Biotechne, Abingdon, UK). Biotinylated bovine serum albumin (bt-BSA) was from Thermo Fisher Scientific, and biotinylated oligonucleotide 80 -mer was synthesized by Eurofins Genomics (Ebersberg, Germany). Finally, biotinylated $\mathrm{VEGF}_{95}\left(\mathrm{bt}-\mathrm{VEGF}_{95}\right)$ and biotinylated peptides A and $\mathrm{B}$ (AC-14 and LW146-2, respectively) were homemade.

Nomenclature. The following terms will be used throughout this manuscript to identify the different types of beads:

- Nongrafted beads are the naked beads

- Saturated beads are the beads totally covered with any ligand 
- $\mathrm{X} \%$ saturated beads are the beads partially covered with any ligand

- Saturation rate (saturated rate) is the percentage of bead surface covered by the ligand

- Binding capacity is the inflection point in the saturation curves

Grafting of Streptavidin-Magnetic Beads. Dynabeads M-280 Streptavidin were functionalized with different biotinylated biomolecules as described in the provider datasheet. $^{24}$ Typically, for functionalization of $0.12 \mathrm{mg}$ of MBs, the beads were resuspended in the vial (vortexed for 30 $\mathrm{s})$, and then $12 \mu \mathrm{L}$ of stock solution was transferred to a tube. MBs were washed two times with $200 \mu \mathrm{L}$ of PBS and resuspended at a concentration of $10 \mathrm{mg} / \mathrm{mL}$ in PBS. The calculated amount of the biotinylated molecule in PBS was added to the $\mathrm{MB}$ and incubated for $1 \mathrm{~h}$ at room temperature using gentle rotation $(300 \mathrm{rpm})$. Grafted beads were washed four times with $300 \mu \mathrm{L}$ of PBS/0.1\% BSA (w/v) and dispersed at the initial concentration of $\mathrm{MB}(10 \mathrm{mg} / \mathrm{mL})$ in $\mathrm{PBS} / 0.1 \%$ BSA and stored at $4{ }^{\circ} \mathrm{C}$ until use.

Consecutive Grafting of Streptavidin-Magnetic Beads. The experimental procedure for consecutive grafting of $\mathrm{MB}$ was similar to the procedure used for grafting (see previous section). In practice, $12 \mu \mathrm{L}$ of $10 \mathrm{mg} / \mathrm{mL} \mathrm{MB}$ was first grafted with different molecules (i.e., biotin, bt-VEGF95, and bt-BSA) at different concentrations. After incubation, supernatants were taken and used to perform a second grafting of another fresh $12 \mu \mathrm{L}$ of $10 \mathrm{mg} / \mathrm{mL} \mathrm{MB}$. After each incubation, $\mathrm{MB}$ were washed and stored at $4{ }^{\circ} \mathrm{C}$ until use.

bt-HRP assay procedure. A white high-binding 96-well plate (Costar 3922) was blocked by adding $200 \mu \mathrm{L} /$ well of blocking buffer (PBS containing 3\% BSA) and incubating for 2 $\mathrm{h}$ at $37{ }^{\circ} \mathrm{C}$. The plate was then washed three times with 200 $\mu \mathrm{L} /$ well of PBS $/ 0.1 \%$ Tween 20 . Afterward, $20 \mu \mathrm{L}$ of the desired diluted grafted $\mathrm{MB}$ (bead dilution $=1: 40$ ) and $100 \mu \mathrm{L}$ of diluted bt-HRP $(15 \mathrm{nM})$ were added to each well $(120 \mu \mathrm{L}$ final volume). Both dilutions were made in PBS/0.05\% Tween 20. After $1 \mathrm{~h}$ of incubation at $37^{\circ} \mathrm{C}$, the plate was washed three times with $200 \mu \mathrm{L} /$ well PBS/0.1\%Tween 20 . Then, 100 $\mu \mathrm{L}$ of chemiluminescent substrate (containing a solution of peroxide and luminol enhancer at $1: 1, \mathrm{v} / \mathrm{v}$ ) was added to each well. The chemiluminescent signal was always measured at the same time ( $5 \mathrm{~min}$ after addition of the chemiluminescent substrate $)$ and relative to a reference signal $\left(S_{\max }\right)$ as shown in eq 1. Measurements were made on an Infinite F200 Pro Microplate Reader from TECAN (Männedorf, Switzerland). It is noteworthy that $\mathrm{MB}$ was fixed on the flat bottom of wells using a 96-well magnet plate both during washing steps and just before measuring the chemiluminescent signal.

For each biotinylated molecule grafted onto $\mathrm{MB}$, the saturated rate (\%) of grafted beads was calculated using eq 1 :

$$
\text { Saturation rate }(\%)=\left[\frac{S_{\max }-S_{i}}{S_{\max }-S_{\text {sat }}}\right] \times 100
$$

where $S_{i}$ is the measured signal for grafted beads, $S_{\max }$ is the maximum signal of the nongrafted beads, and $S_{\text {sat }}$ is the signal of $100 \%$ saturated beads (obtained with a large excess of ligand, approximately 2 times the binding capacity). All experiments were performed in triplicate, unless otherwise indicated.

\section{ASSOCIATED CONTENT}

\section{S Supporting Information}

The Supporting Information is available free of charge on the ACS Publications website at DOI: 10.1021/acs.bioconjchem.8b00347.

\section{Supporting figures (PDF)}

\section{AUTHOR INFORMATION}

\section{Corresponding Author}

*Phone: +33 11537397 44. E-mail: nathalie.eilstein@ parisdescartes.fr.

ORCID $\odot$

Jorge Ruiz Encinar: 0000-0001-6245-5770

Nathalie Gagey-Eilstein: 0000-0003-0185-2080

\section{Author Contributions}

"These authors contributed equally.

\section{Notes}

The authors declare no competing financial interest.

\section{ACKNOWLEDGMENTS}

We would like to acknowledge the financial support provided by the Agence National de la Recherche (ANR-15-CE17-000501) and the Spanish Ministry of Education (CTQ2016-79412P). D.B.-R. acknowledges the Ph.D. grant (BP14-137) from Asturias Regional Government (Spain) and the mobility grant convened by the University of Oviedo and financed by Banco Santander. Paris Descartes University, INSERM, and CNRS are also acknowledged. We would also thank to Dr. J. F. Gaucher, Dr. W-Q. Liu, and Dr. R. Gahoual for kindly providing the biotinylated VEGF95, peptides, and TNF $\alpha$ protein, respectively. Finally, the UMR $8258-\mathrm{CNRS}$ is acknowledged for sharing the TECAN SAFIRE plate-reader.

\section{REFERENCES}

(1) Sapsford, K. E., Tyner, K. M., Dair, B. J., Deschamps, J. R., and Medintz, I. L. (2011) Analyzing nanomaterial bioconjugates: a review of current emerging purification and characterization techniques. Anal. Chem. 83, 4453-4488.

(2) Von Der Kammer, F. P., Ferguson, L., Holden, P. A., Masion, A., Rogers, K. R., Klaine, S. J., Koelmans, A. A., Horne, N., and Unrine, J. M. (2012) Analysis of engineered nanomaterials in complex matrices (environment and biota): general considerations and conceptual case studies. Environ. Toxicol. Chem. 31, 32-49.

(3) Mahmoudi, M., Bertrand, N., Zope, H., and Farokhzad, O. C. (2016) Emerging understanding of the protein corona at the nano-bio interfaces. Nano Today 11, 817-832.

(4) Feliu, N., Pelaz, B., Zhang, Q., del Pino, P., Nyström, A., and Parak, W. J. (2016) Nanoparticle Dosage-A Nontrivial Task of Utmost Importance for Quantitative Nanosafety Research. WIREs Nanomed. Nanobiotechnol. 8, 479-492.

(5) Dobrovolskaia, M. A., Patri, A. K., Zheng, J., Clogston, J. D., Ayub, N., Aggarwal, P., Neun, B. W., Hall, J. B., and McNeil, S. E. (2009) Interaction of colloidal gold nanoparticles with human blood: effects on particle size and analysis of plasma protein binding profiles. Nanomedicine 5 (2), 106-117.

(6) Tsai, D.-H., Davila-Morris, M., DelRio, F. W., Guha, S., Zachariah, M. R., and Hackley, V. A. (2011) Quantitative determination of competitive molecular adsorption on gold nanoparticles using attenuated total reflectance-fourier transform infrared spectroscopy. Langmuir 27, 9302-9313.

(7) Hinterwirth, H., Kappel, S., Waitz, T., Prohaska, T., Lindner, W., and Lämmerhofer, M. (2013) Quantifying thiol ligand density of selfassembled monolayers on gold nanoparticles by inductively coupled plasma-mass spectrometry. ACS Nano 7 (2), 1129-1136. 
(8) Xia, X., Yang, M., Wang, Y., Zheng, Y., Li, Q., Chen, J., and Xia, Y. (2012) Quantifying the coverage density of poly(ethylene glycol) chains on the surface of gold nanostructures. ACS Nano 6 (1), 512522.

(9) Bouzas-Ramos, D., Menéndez-Miranda, M., Costa-Fernández, J. M., Encinar, J. R., and Sanz-Medel, A. (2016) Precise determination of the nanoparticle concentration and ligand density of engineered water-soluble $\mathrm{HgSe}$ fluorescent nanoparticles. RSC Adv. 6, 1996419972.

(10) Garcia-Cortes, M., Sotelo González, E., Fernández-Argüelles, M. T., Encinar, J. R., Costa-Fernández, J. M., and Sanz-Medel, A. (2017) Capping of Mn-doped ZnS quantum dots with DHLA for their stabilization in aqueous media: determination of the nanoparticle number concentration and surface ligand density. Langmuir 33, 6333-6341.

(11) Elzey, S., Tsai, D.-H., Rabb, S. A., Yu, L. L., Winchester, M. R., and Hackley, V. A. (2012) Quantification of ligand packing density on gold nanoparticles using ICP-OES. Anal. Bioanal. Chem. 403, 145149.

(12) Huber, A., Behnke, T., Würth, C., Jaeger, C., and ReschGenger, U. (2012) Spectroscopic characterization of coumarinstained beads: quantification of the number of fluorophores per particle with solid-state 19F-NMR and measurement of absolute fluorescence quantum yields. Anal. Chem. 84, 3654-3661.

(13) Sebby, K. B., and Mansfield, E. (2015) Determination of the surface density of polyethylene glycol on gold nanoparticles by use of microscale thermogravimetric analysis. Anal. Bioanal. Chem. 407, 2913-2922.

(14) Torelli, M. D., Putans, R. A., Tan, Y., Lohse, S. E., Murphy, C. J., and Hamers, R. J. (2015) Quantitative determination of ligand densities on nanomaterials by $\mathrm{X}$-ray photoelectron spectroscopy. ACS Appl. Mater. Interfaces 7, 1720-1725.

(15) Ndolomingo, M. J., and Meijboom, R. (2016) Determination of the surface area and sizes of supported copper nanoparticles through organothiol adsorption-chemisorption. Appl. Surf. Sci. 390, 224-235.

(16) Walkey, C., Olsen, J. B., Song, F., Liu, R., Guo, H., Olsen, D. W. H., Cohen, Y., Emili, A., and Chan, W. C. W. (2014) Protein corona fingerprinting predicts the cellular interaction of gold and silver nanoparticles. ACS Nano 8 (3), 2439-2455.

(17) Ansar, S. M., Haputhanthri, R., Edmonds, B., Liu, D., Yu, L., Sygula, A., and Zhang, D. (2011) Determination of the binding affinity, packing, and conformation of thiolate and thione ligands on gold nanoparticles. J. Phys. Chem. C 115, 653-660.

(18) Dorgan, L., Magnotti, R., Hou, J., Engle, T., Ruley, K., and Shull, B. (1999) Methods to determine biotin-binding capacity of streptavidin-coated magnetic particles. J. Magn. Magn. Mater. 194 (1), $69-75$.

(19) Van der Valk, A. M., Howbrook, D. N., O'Shaughnessy, M. C., Sarker, D. K., Baker, S. C., Louwrier, A., and Lloyd, A. W. (2003) Methods to quantify the biotin-binding capacity of streptavidin-coated polypropylene PCR plates. Biotechnol. Lett. 25 (16), 1325-1328.

(20) Mittal, R., and Bruchez, M. P. (2011) Biotin-4-fluorescein based fluorescence quenching assay for determination of biotin binding capacity of streptavidin conjugated quantum dots. Bioconjugate Chem. 22, 362-368.

(21) Jamshaid, T., Neto, E. T. T., Eissa, M. M., Zine, N., Kunita, M. H., El-Salhi, A. E., and Elaissari, A. (2016) Magnetic particles: from preparation to lab-on-a-chip, biosensors, microsystems and microfluidics applications. TrAC, Trends Anal. Chem. 79, 344-362.

(22) Aseri, A., Garg, S. K., Nayak, A., Trivedi, S. K., and Ahsan, J. (2015) Magnetic nanoparticles: magnetic nano-technology using biomedical applications and future prospects. Int. J. Pharm. Sci. Rev. Res. 31 (2), 119-131.

(23) Piran, U., and Riordan, W. J. (1990) Dissociation rate constant of biotin-streptavidin complex. J. Immunol. Methods 133 (1), 141143.

(24) Dynabeads M-280 Streptavidin. (2015) https://assets. thermofisher.com/TFS-Assets/LSG/manuals/MAN0014017 Dynabeads_M280_Streptavidin_UG.pdf.
(25) Rao, A. N., and Grainger, D. W. (2014) Biophysical properties of nucleic acids at surfaces relevant to microarray performance. Biomater. Sci. 2, 436-471.

(26) Safarik, I., Horska, J., Pospiskova, K., and Safarikova, M. (2012) Magnetic techniques for the detection and determination of xenobiotics and cells in water. Anal. Bioanal. Chem. 404, 1257-1273. 